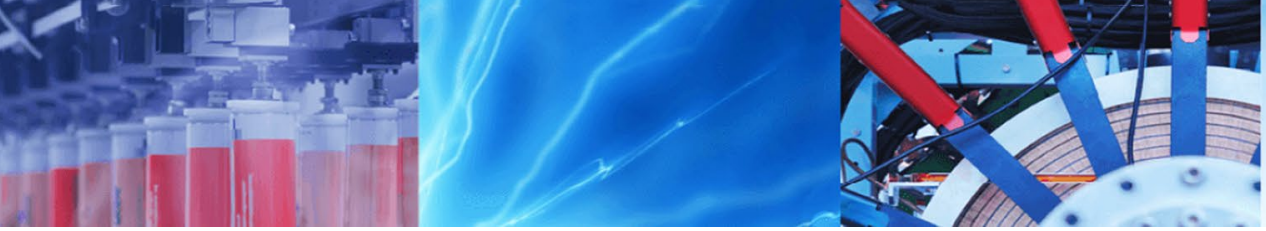

Research Article

\title{
Three-dimensional graphene-wrapped porous carbon/sulfur composite for cathode of lithium-sulfur battery
}

\author{
Yating Wang ${ }^{1} \cdot$ Bin Liu $^{1} \cdot$ Wei Zhang ${ }^{1}$. Changhong Shao ${ }^{1}$. Daoyun Lan ${ }^{1} \cdot$ Xiaofeng Qu ${ }^{1} \cdot$ Rongfeng Chen $^{1}$. \\ Weiqing Zhang ${ }^{1} \cdot$ Weimin Zhao $^{2}$. Jun Liu ${ }^{1} \cdot$ Yinghe Zhang $^{3} \cdot$ Zhicong Shi $^{1}$ (i)
}

Received: 29 April 2020 / Accepted: 12 June 2020 / Published online: 23 June 2020

(c) Springer Nature Switzerland AG 2020

\begin{abstract}
Lithium-sulfur battery with high theoretical capacity becomes the subject of recent attention. Its commercial progress is impeded by its poor electrical conductivity and high dissolubility of intermediate products in organic electrolyte. In the present work, we report a novel three-dimensional graphene-wrapped porous carbon (3D-G) to accommodate sulfur, which consists of outside highly stable interconnected graphene-like sheets and inner activated porous carbon. The 3D-G was synthesized from cheap polyacrylic acid cation-exchange resin, which introduces nickel ions as catalysts in the carbonization process, as a cost effective, facile and simple method. This 3D-G showed hierarchical pores: $0.7 \mathrm{~nm}$ micropores and macropores, providing a large specific surface area of $1375.08 \mathrm{~m}^{2} \mathrm{~g}^{-1}$. Benefiting from this unique structure, the 3D-G performed well as host materials to achieve a high sulfur content (75.4 wt\%). Such a 3D-G@S composite exhibits capacity-fading rate as low as $0.28 \%$ per cycle over 100 cycles at $0.1 \mathrm{C}$, and good cyclability at various cycling rates $(0.1-1 \mathrm{C})$.
\end{abstract}

Keywords Three-dimensional graphene · Porous carbon · Lithium sulfur battery · Polyacrylic acid cation-exchange resin

\section{Introduction}

The increasing markets of electrical vehicles, cell phones and new electronic devices make it urgently desirable to develop and optimize rechargeable batteries with high energy density [1-4]. Presently, rechargeable lithium ion batteries (LIB) hold the dominant share in the markets for portable electronic devices. Nevertheless, the energy and power density of LIBs still need significant improvements in order to support electric cars. In such a situation, developing higher level battery systems with higher energy density and safety through new configurations and electrode materials are of tremendous significance [5-11].

Sulfur has a set of advantages, viz., cheap, abundant and environment friendly [12]. Especially, sulfur with theoretical capacity of $1672 \mathrm{mAh} \mathrm{g}^{-1}$, almost ten times the capacity of traditional LIBs, is a charming cathode material [13-15]. Howbeit, the commercial progress of $\mathrm{Li}-\mathrm{S}$ battery is impeded by its poor electrical conductivity, accompanied by low utilization of elemental sulfur. Another big issue for Li-S battery is the high dissolubility of the intermediate products called lithium polysulfides $\left(\mathrm{Li}_{2} \mathrm{~S}_{x^{\prime}} 4 \leq \mathrm{x} \leq 8\right)$, which can reduce the cycle stability of battery [16-19]. The product $\left(\mathrm{Li}_{2} \mathrm{~S}_{2}\right.$ and $\left.\mathrm{Li}_{2} \mathrm{~S}\right)$ which deposited uncontrollably are acknowledged as another important reason for accelerated capacity deterioration and low Coulombic efficiency in Li-S batteries [20-22]. These deficiencies remarkably undermine energy density of Li-S battery.

To eliminate these shortcomings, substantial efforts have been made to design host materials such as carbon

$\square$ Jun Liu, junliu23@gdut.edu.cn; $\bowtie$ Yinghe Zhang, Caroline.w.2006@gmail.com; $₫$ Zhicong Shi, zhicong@gdut.edu.cn| ${ }^{1}$ Guangdong Provincial Engineering Technology Research Centre for New Energy Materials and Devices, School of Materials and Energy, Guangdong University of Technology, Guangzhou 510006, People's Republic of China. ${ }^{2}$ College of Chemical Engineering and Safety, Binzhou University, Binzhou 256503, People's Republic of China. ${ }^{3}$ Nanotechnology Department, Helmholtz Association, 21502 Hamburg, Germany. 
materials $[5,11,23-38]$ and nanometal oxides [6]. These approaches have been considered to boost the electrical conductivity of sulfur materials and confine soluble polysulfides. For all that, the Li-S battery has not been available in practical application till today, because most of the reported electrodes have low areal mass loading, unavoidably decreasing the real capacity of the Li-S battery systems. Up till now, many efforts have been made to increase the areal mass loading, such as introducing 3D structure hosts $[11,26]$. 3D graphene oxides with abundant porous structure have been applied as host materials to achieve high areal mass loading and excellent contact with sulfur for the sake of better electron transport. Particularly, Rehman et al. announced an interconnected and vertically aligned porous carbon nanosheets with good electrical conductivity, which show promising electrochemical properties as a sulfur host [11]. All in all, exploring efficient approaches to encapsulate sulfur into 3D carbon matrix has a bright prospect $[24,25]$.

Here, we introduce a simple method to synthesize three-dimensional porous graphene-wrapped porous carbon using PAA cation-exchange resin as raw material with nickel ions as catalysts. A melting diffusion approach was used to incorporate sulfur into $3 D-G$ porous structure. The amount of sulfur mass can be strictly controlled by the impregnation approach. The key feature of 3D-G@S is that they have traits of both porous carbon and graphene: (a) 3D-G possesses high surface area of $1375.08 \mathrm{~m}^{2} \mathrm{~g}^{-1}$, which in its unique porous structure allows high sulfur loading for improving the energy density; (b) in addition, it can buffer the volume variation during the conversion processes between sulfur and its discharged products, preserve the integrity of the electrode structure, and boost the cycling performance of the batteries; (c) the graphenelike structure on the surface confines sulfur within the cathode and suppresses the free movement of the polysulfide species; (d) 3D-G helps to improve the electrical conductivity, decrease surface impedance and boost rate capability.

\section{Materials and methods}

Sodium hydroxide (AR), potassium hydroxide (AR), Nickel(II) acetate tetrahydrate (AR) and sulfur powders (AR) were bought from Aladdin. Polyacrylic acid cationexchange resin was bought from Shanghai Hualing Company. Ethanol (AR) was bought from Tianjin Damao Company. All the chemicals were used with no further modification.

SN Applied Sciences

\subsection{Material synthesis}

\subsubsection{Synthesis of 3D-G}

Polyacrylic acid cation-exchange resin ( $500 \mathrm{~g}$ ) was initially cleaned using deionized water, and immersed in $1 \mathrm{M} \mathrm{HCl}$ solution for more than $10 \mathrm{~h}$. It was then washed with deionized water and dried at $70^{\circ} \mathrm{C}$ in blast drying oven. The prepared hydrogen ion-exchange resin was immersed in $1 \mathrm{M} \mathrm{NaOH}$ solution for $10 \mathrm{~h}$, which were washed and dried as above. $10 \mathrm{~g}$ obtained powders were added into $100 \mathrm{ml}$ nickel acetate solution $\left(0.05 \mathrm{~mol} \mathrm{~L}^{-1}\right)$ to get nickel ion-exchange resin.

The $\mathrm{KOH}$-ethanol solution was prepared by dissolving $40 \mathrm{~g} \mathrm{KOH}$ in $400 \mathrm{ml}$ anhydrous ethanol. The as-synthesized resin was added in the $\mathrm{KOH}$-ethanol solution, then stirred and heated at $80^{\circ} \mathrm{C}$ in water bath until complete vaporization of ethanol to form the 3D-G precursor. These precursor materials were kept at room temperature overnight, and then placed in the $70^{\circ} \mathrm{C}$ blast drying oven for $6 \mathrm{~h}$. After drying, the powder was heated for $2 \mathrm{~h}$ at $850^{\circ} \mathrm{C}$ in $\mathrm{Ar}$ atmosphere with the heating-rate of $2{ }^{\circ} \mathrm{C} \mathrm{min}^{-1}$ to produce the 3D-G material. These 3D-G materials were dissolved in $3 \mathrm{~mol} \mathrm{~L}^{-1} \mathrm{HCl}$ in order to remove the residual $\mathrm{KOH}$ until neutral, and finally dried at $100^{\circ} \mathrm{C}$ for $12 \mathrm{~h}$ under vacuum.

\subsubsection{Synthesis of 3D-G @S}

The as-prepared 3D-G and the elemental sulfur were mixed together in weight ratios of 1:4. Then sulfur/3D$\mathrm{G}$ mixtures were put into a sealed vessel and heated at $155^{\circ} \mathrm{C}$ for $12 \mathrm{~h}$ to let the melting sulfur absorbed into the pores of 3D-G via diffusion.

\subsection{Material characterization}

The characterization of the 3D-G and 3D-G@S composites were performed by thermogravimetric analyzer (TGA, TA Instrument SDT2960), Brunauer-Emmett-Teller (BET, ASAP 2020), X-ray diffraction (XRD, RIGAKUD/max-2500), and scanning electron microscopy (SEM, HITACHI S-3500 N).

\subsection{Electrochemical measurements}

The electrochemical performance of 3D-G@S was assessed by CR2016-type coin cells. The counter electrode is a lithium foil and the separator is a polypropylene microporous film (Celgard 2300). The cathode electrode was fabricated by blending 3D-G@S with PVDF binder and conducting additive (Super $P$ ) following the weight ratio of $8: 1: 1$. In the cathode electrode, the mass loading of sulfur was 
about $3 \mathrm{mg} \mathrm{cm}^{-2}$. And the electrolyte was composed of 1.0 M LiTFSI (lithiumbis(trifluoromethanesulfonyl)imide) in 1,3-dioxolane(DOL) and 1,2-dimethoxyethane (DME) (1:1 in volume ratio, with $1.0 \mathrm{wt} \% \mathrm{LiNO}_{3}$ as additive). $0.1 \mathrm{ml}$ electrolyte was used in a coin cell. The cells were tested by Neware SBT7.5 in the voltage scope from 1.5 to $3.0 \mathrm{~V}$ at various current rates $\left(1 \mathrm{C}=1675 \mathrm{~mA} \mathrm{~g}^{-1}\right)$. Cyclic voltammetry (CV) measurements over the potential range of $1.5-3 \mathrm{~V}$ at $0.1 \mathrm{mV} / \mathrm{s}$ scanning rate and the electrochemical impedance spectrum (EIS) tests with a frequency from $100 \mathrm{kHz}$ to $0.1 \mathrm{~Hz}$ were carried out on an electrochemical workstation (Solartron 1287/1260).

\section{Results and Discussion}

Figure 1 demonstrates the synthetic process of 3D-G. Firstly, polyacrylic acid cation-exchange resin was treated with $\mathrm{HCl}$ solution for more than $10 \mathrm{~h}$ to produce hydrogen ion-exchange resin. The prepared hydrogen ion-exchange resin was immersed in $1 \mathrm{M} \mathrm{NaOH}$ solution for $10 \mathrm{~h}$ to obtain sodium ion-exchange resin, which was then immersed in nickel acetate solution to get nickel ion-exchange resin. At high temperature nickel works as catalytic site to aid the growth of carbon. In the next step, direct carbonization of nickel ion-exchange resin with $\mathrm{KOH}$ as activator produces highly interconnected and porous 3D-G.

The morphology of the pure resin carbon and 3D-G were characterized by FE-SEM. Figure $2 a, b$ are the SEM images of pure resin carbon with different magnifications and exhibit that big pieces of granular carbon join together. Figure $2 c$, d show that the as-prepared 3D-G possesses white fungus-like structure with wrinkled graphene linked together.

The formation of the special 3D-G structure can be described by the cooperation effect of $\mathrm{Ni}$ atoms and $\mathrm{KOH}$. It has been proclaimed that $\mathrm{Ni}$ and $\mathrm{Cu}$ perform as the most promising graphene catalytic metals via a dissolution-precipitation mechanism [39]. Blakely and coauthors have operated extensive studies on the formation of carbon films. They found that a single layer graphite forms first through carbon segregation, followed by precipitation of more carbon to grow graphite $[39,40]$.
Fig. 1 Schematics illustration on synthesis method for 3D-G polyacrylic acid cation exchange resin

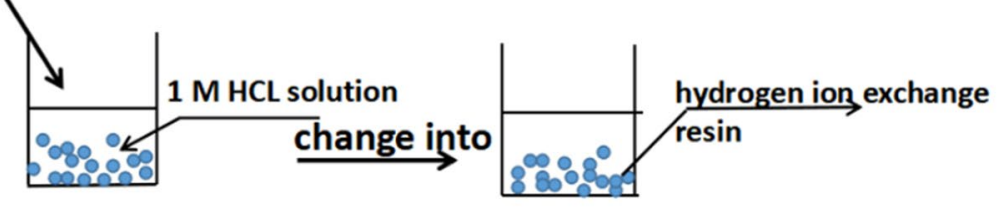

hydrogen ion exchange resin

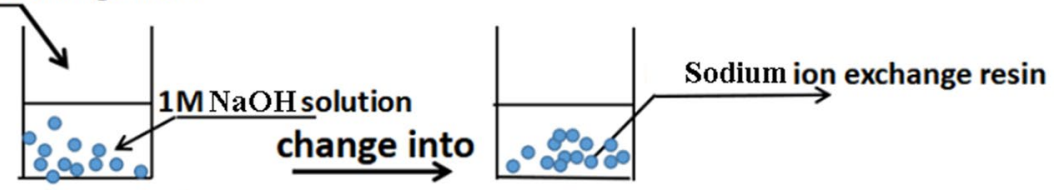

Sodium on exchange resin

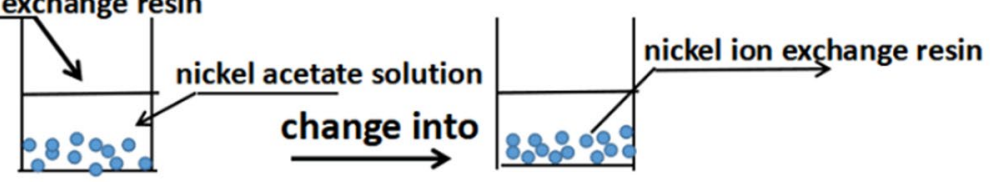

Nickel ion exchange resin
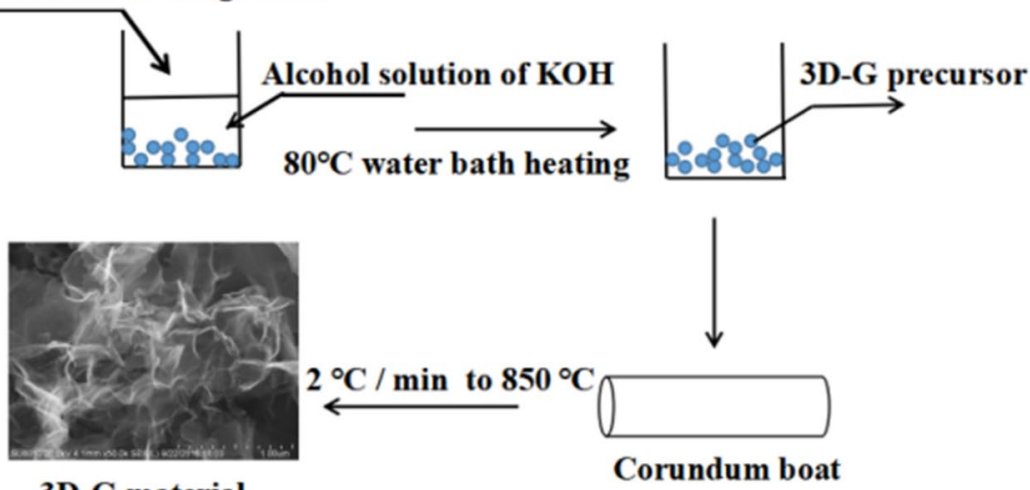

3D-G material 

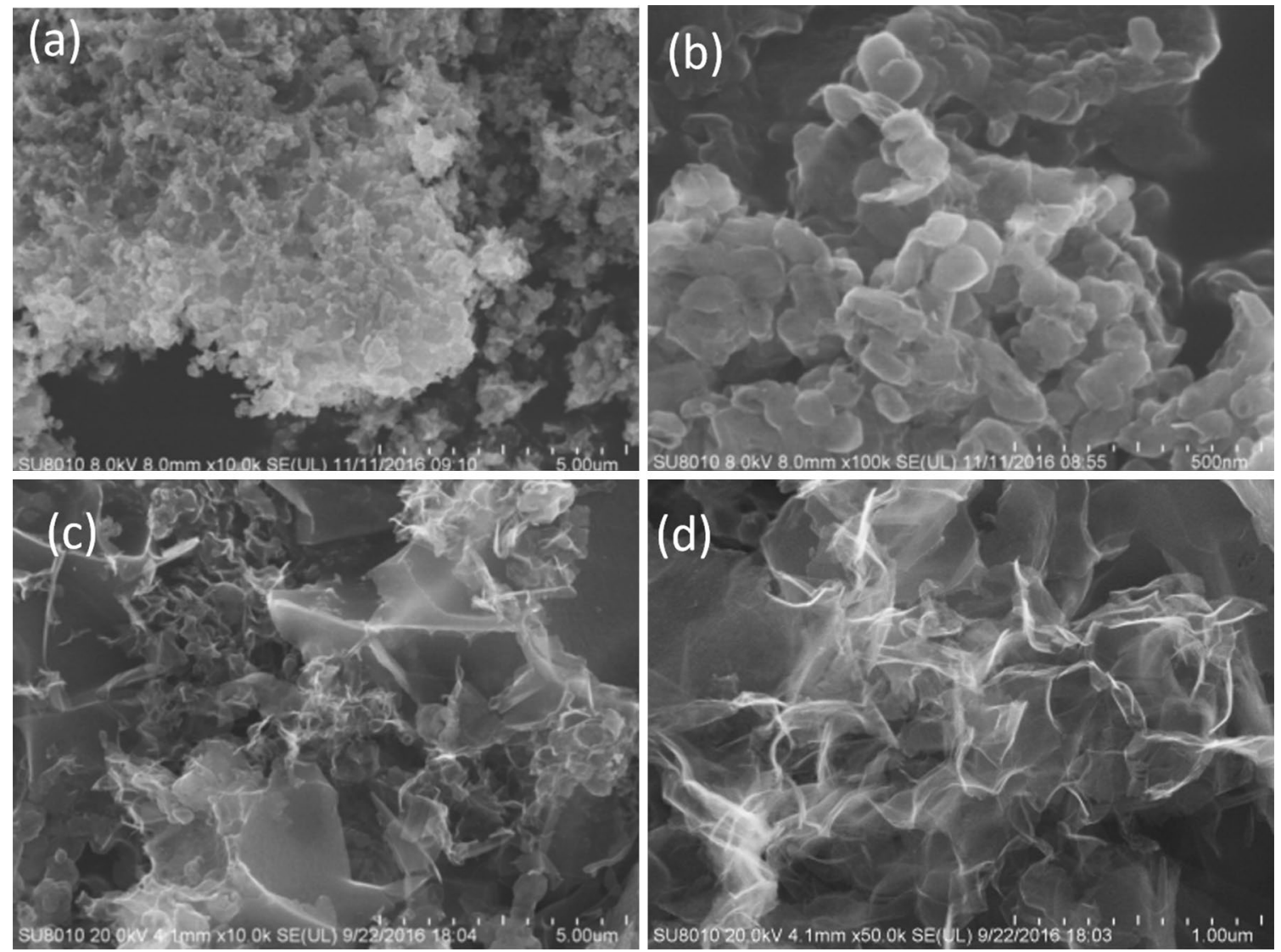

Fig. 2 The SEM images for pure resin carbon: a low resolution; $\mathbf{b}$ high resolution; SEM images for 3D-G: $\mathbf{c}$ low resolution; $\mathbf{d}$ high resolution

Firstly potassium carbonate forms (at $\leq 600^{\circ} \mathrm{C}$ ), which is then decomposed to $\mathrm{K}_{2} \mathrm{O}$ and $\mathrm{CO}_{2}$ (at $\leq 700^{\circ} \mathrm{C}$ ). Finally, at $\mathrm{T} \leq 800{ }^{\circ} \mathrm{C}$, the produced $\mathrm{CO}_{2}$ reacts with carbon to produce porosity at high temperature [41]. Thanks to $\mathrm{Ni}$ catalysis and $\mathrm{KOH}$ activation, 3D-G has surface area as high as $1375.08 \mathrm{~m}^{2} \mathrm{~g}^{-1}$, which can offer enough space for high loading amount of sulfur to improve the energy density of lithium-sulfur battery. Unlike the conventional twodimensional porous carbon sheets, $3 D-G$ is the assembly of inner porous carbon and outer interconnected carbon nanosheets. This unique structure serves as a cage for trapping polysulfide anions and is highly important for alleviating the dissolution of polysulfides during battery cycling.

For further confirmation, X-ray diffraction was conducted. XRD patterns for pure S, 3D-G, 3D-G@S, and resin carbon are shown in Fig. 3a. Pure resin carbon has obvious sharp peaks at $26^{\circ}$ and $43^{\circ}$, which correspond to the graphite phase (002) and (110) crystal plane, indicating that carbon generated from pure resin has good graphitic carbon structure. However, after dealing with the nickel catalytic reaction and $\mathrm{KOH}$ activation, the sharp peak at $2 \theta=26^{\circ}$ shifts to the left, demonstrating that the 3D-G spacing has become broader, which is mainly due to the catalytic function of nickel ions. In the forming process of 3D-G, nickel atom reacts with carbon to form nickel carbide at low temperature. When the temperature rises to $500^{\circ} \mathrm{C}$, nickel carbide is further decomposed to nickel and a stable crystal lattice of carbon, which accelerate the rearrangement and expansion of carbon layer. In addition, peak for crystalline sulfur is not observed in 3D-G@S, verifying that sulfur is completely distributed within the porous carbon structure and graphene sheets.

Nitrogen BET adsorption were used to measure the specific surface area of 3D-G and 3D-G@S composites. The nitrogen adsorption-desorption isotherms of 3D-G and 3D-G@S, are displayed in Fig. 3b, respectively. The shape of 3D-G isotherm is described as type I and indicates the presence of pore sizes varying from micropores to macropores. The quick increase at a comparatively low pressure of $P / P_{0}\left(0 \leq P / P_{0} \leq 0.1\right.$, where $P$ and $P_{0}$, correspond, respectively, to the equilibrium and saturation pressure for $\mathrm{N}_{2}$ at $77 \mathrm{~K}$ ), indicates the existence of micropores, which is based on the behaviors of nitrogen filling in micropores during the adsorption process. In the area $\mathrm{P} / \mathrm{P}_{0} \geq 0.1$, the gas adsorption slowly increases, which reveals that the material has less mesoporous structure. As the pressure

\section{SN Applied Sciences


(a)

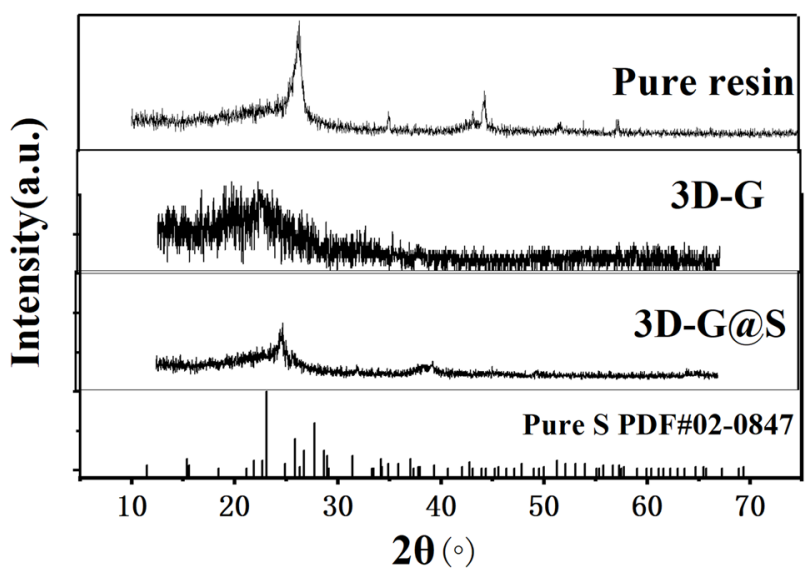

(c)

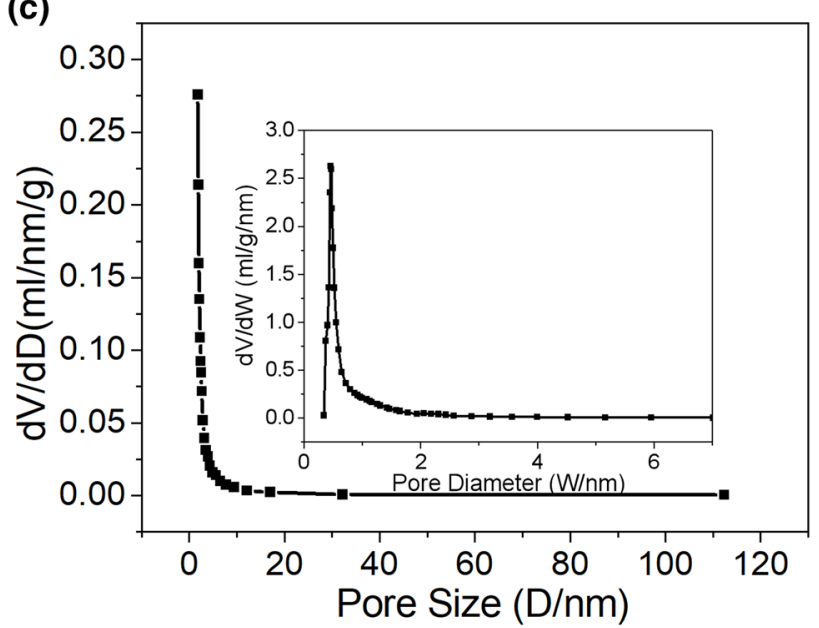

(b)

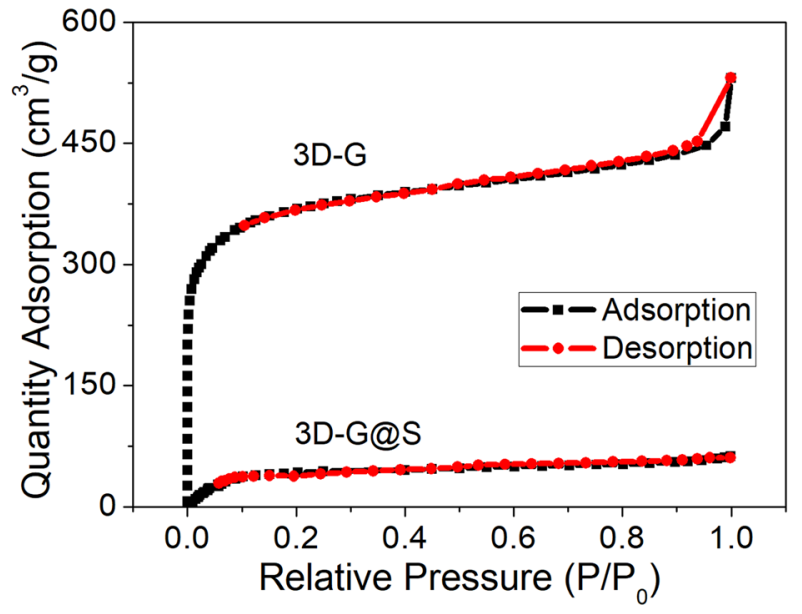

(d)

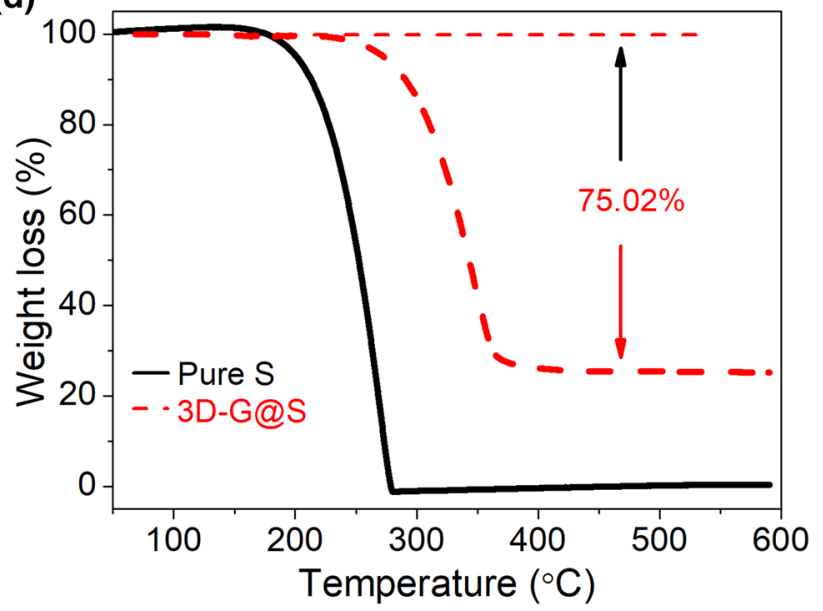

Fig. 3 a XRD patterns for 3D-G, 3D-G@S and resin carbon; b nitrogen adsorption-desorption for 3D-G and 3D-G@S; c the distribution of pore size for 3D-G; d TGA profile image of pure S and 3D-G@S

Table 1 Comparison between 3D-G and 3D-G@S

\begin{tabular}{lcl}
\hline Sample & Specific surface area $\left(\mathrm{cm}^{2} / \mathrm{g}\right)$ & $\begin{array}{l}\text { Total pore } \\
\text { volume } \\
\left(\mathrm{cm}^{3} / \mathrm{g}\right)\end{array}$ \\
\hline 3D-G & 1375.08 & 0.82 \\
3D-G@S & 128.41 & 0.04 \\
\hline
\end{tabular}

reaches to 1.0, a final increasing tail means the existence of macropores. It can be seen in Fig. 3c that 3D-G was primarily composed of micropores with a narrow pore-size distribution of about $0.7 \mathrm{~nm}$. Compared with 3D-G, the gas adsorption curve of the 3D-G@S isotherm slowly increases, indicating that the micropores of the 3D-G material are almost filled with sulfur. As the relative pressure is close to 1.0, the slope of the curve is nearly not going up, which means that the macropores have also been stuffed with sulfur. It can be seen from Table 1 that the specific surface area of the materials decreased from 1375.08 to $128.41 \mathrm{~m}^{2} \mathrm{~g}^{-1}$, and the overall pore volume decreased from 0.82 to $0.04 \mathrm{~cm}^{2} \mathrm{~g}^{-1}$, which supports that the encapsulated sulfur is well dispersed into the pores of 3D-G.

In Fig. 3d, TGA curve shows that pure sulfur begins to vaporize from $\approx 180^{\circ} \mathrm{C}$ and stops at $\approx 300^{\circ} \mathrm{C}$, while $3 \mathrm{D}$-G@S begins to vaporize at $\approx 250^{\circ} \mathrm{C}$ and completes at $\approx 360^{\circ} \mathrm{C}$. The contents of sulfur in the composites are calculated to be $75.02 \%$. Pure $S$ has a lower sulfur vaporizing temperature than 3D-G@S because the interaction between sulfur and carbon-based matrix makes the evaporation of sulfur more difficult.

After confirmation from TGA that sulfur is well dispersed into the pores of 3D-G, now it is important to emphasize the morphology of materials. Figure $4 a$ displays the morphology of large bulk of pure sulfur. Figure $4 b, c$ compare the SEM images of 3D-G and 3D-G@S. It can be seen that 


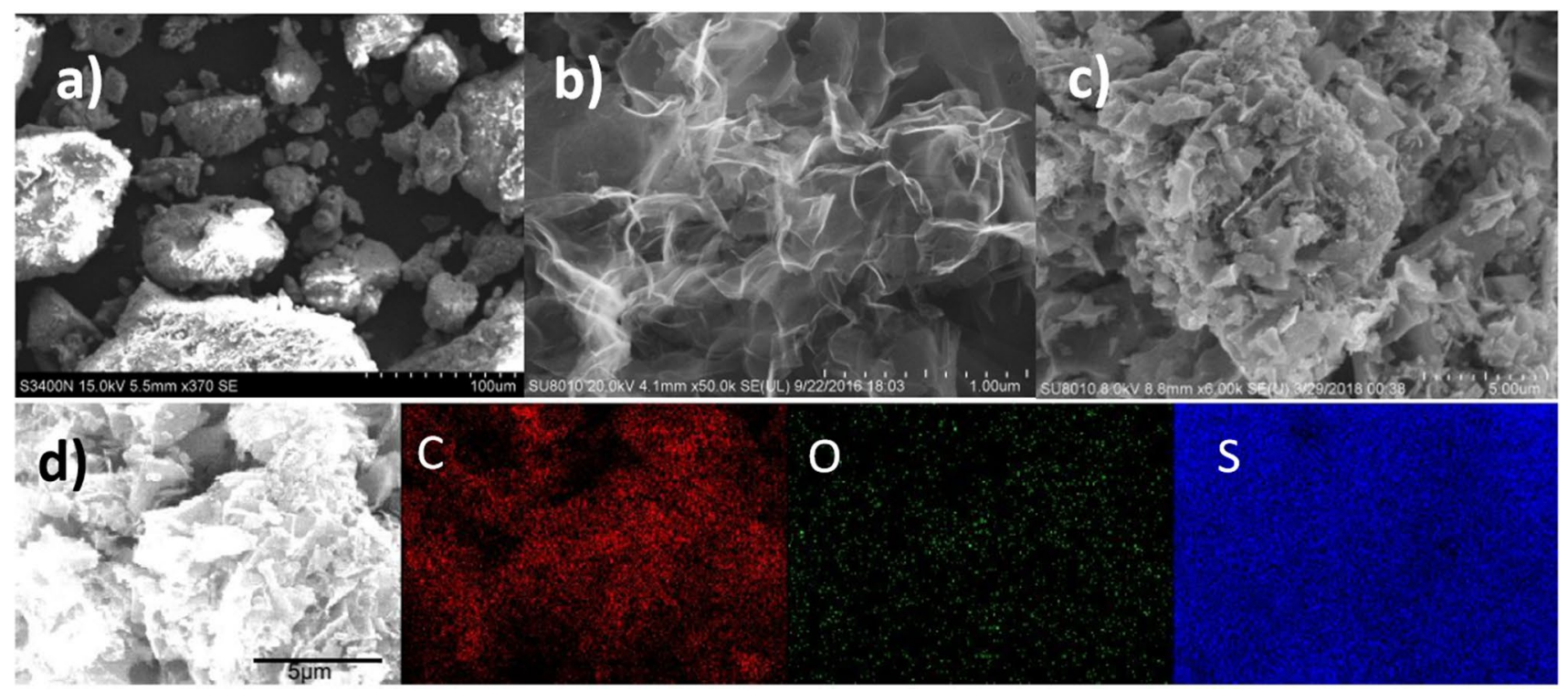

Fig. 4 The SEM images of a pure S; b 3D-G; c 3D-G@S; $\mathbf{d}$ the element mapping of 3D-G@S

graphene-like lamellar structure on the original 3D-G has disappeared after sulfur loading. It indicates the uniform encapsulation of sulfur within the graphene lamellar or graphene lamellar stacked holes, which agrees with the XRD diffraction analysis. The element mapping in Fig. 4d shows that 3D-G@S contains some oxygen element in addition to sulfur and carbon element.Therefore, 3D-G@S also consists of small amount of oxygen-containing functional groups, which could help to offer chemical adsorption to polysulfide ions.

CV curves of 3D-G@S and pure sulfur were compared in Fig. 5a. During the first negative scan of 3D-G@S, it displays two main reductive peaks at around $2.25 \mathrm{~V}$ and $2.04 \mathrm{~V}$. The first reduction peak at about $2.28 \mathrm{~V}$ corresponds to the conversion of $\mathrm{S}_{8}$ to long chain lithium polysulfides $\left(\mathrm{Li}_{2} \mathrm{~S}_{\mathrm{n}}\right.$, $4 \leq n \leq 8$ ) [16]. The second peak at $2.04 \mathrm{~V}$ originates from the reduction of lithium polysulfide to $\mathrm{Li}_{2} \mathrm{~S}_{2}$ and finally to $\mathrm{Li}_{2} \mathrm{~S}$. At $2.46 \mathrm{~V}$, an anodic peak can be assigned to the oxidation of $\mathrm{Li}_{2} \mathrm{~S}_{2} / \mathrm{Li}_{2} \mathrm{~S}$ to form sulfur. During the second negative scan of 3D-G@S, the reductive peaks were noticed to positively shift from 2.25 to $2.30 \mathrm{~V}$, indicating a reduced polarization in the process. As comparison, in the first cycle of pure sulfur, an oxidation peak was observed at $2.65 \mathrm{~V}$, which was negatively shifted to $2.5 \mathrm{~V}$ in the second circle. The polarization and irreversible capacity loss of pure sulfur are obviously larger than 3D-G@S composite. Furthermore, the CV curve of the 3D-G@S composite in the first two circles is better coincident than pure $S$, which indicates that the material has a better reversible performance.

Figure 5b, c show the discharge/charge profiles of pure $S$ in the first 5 cycles and the first 200 cycles at $0.1 \mathrm{C}$ rate, respectively. Pure $S$ exhibits an initial discharge capacity $934.2 \mathrm{mAh} \mathrm{g}^{-1}$ and a capacity of $291.9 \mathrm{mAh} \mathrm{g}^{-1}$ in the 200 th cycle at $0.1 \mathrm{C}$. In addition, the large potential difference between the discharge plateau and charge plateau suggested that the polarization of pure $\mathrm{S}$ electrode is large, which is consistent with Fig. 5a. Figure $5 d$, e are the discharge/charge profiles of 3D-G@S in the first 5 cycles and 200 cycles at $0.1 \mathrm{C}$ rate, respectively. It shows that 3D-G@S composite material exhibits an initial discharge capacity as $1066.4 \mathrm{mAh} \mathrm{g}^{-1}$ at $0.1 \mathrm{C}$. As the cycle went on, capacity decayed slowly.3D-G@S showed a specific capacity of $701.3 \mathrm{mAh} \mathrm{g}^{-1}$ with capacity retention of $55.6 \%$ after 200 cycles. In addition, 3D-G@S composite material shows an obvious activation process. It is found that capacity of 3D-G@S rose in the initial several cycles. The possible reason for this phenomenon is that in the initial several cycles, the wettability between the electrolyte and sulfur is not good as the channels in 3D-G carbon are blocked by sulfur materials. In this case, some sulfur does not react with the electrolyte at the beginning. After several cycles, the electrolyte contacts with sulfur materials thoroughly due to formation of polysulfides and the dredged inner pathway.

Table 2 is the redox potential of the first two CV cycles for pure S and 3D-G@S.3D-G@S has lower oxidation peak potential than pure $S$, which means lower polarization in 3D-G@S.

Figure 6a compares the cycling stability between 3D-G@S and pure S at 0.1 C. 3D-G@S has initial discharge capacity of $1066.4 \mathrm{mAh} \mathrm{g}^{-1}$, almost $98.7 \%$ coulombic efficiency and $63.7 \%$ utilization rate of the active materials. Capacity retention ratio was $54.7 \%$ after 200 cycles. 
(a)

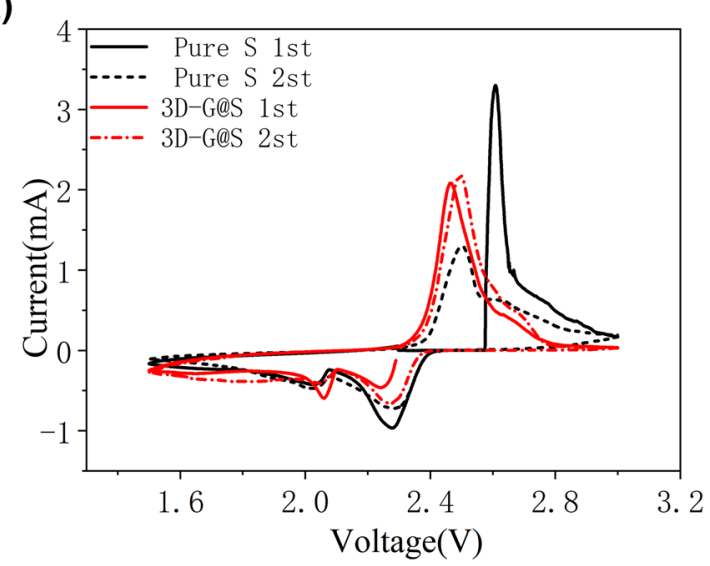

(c)

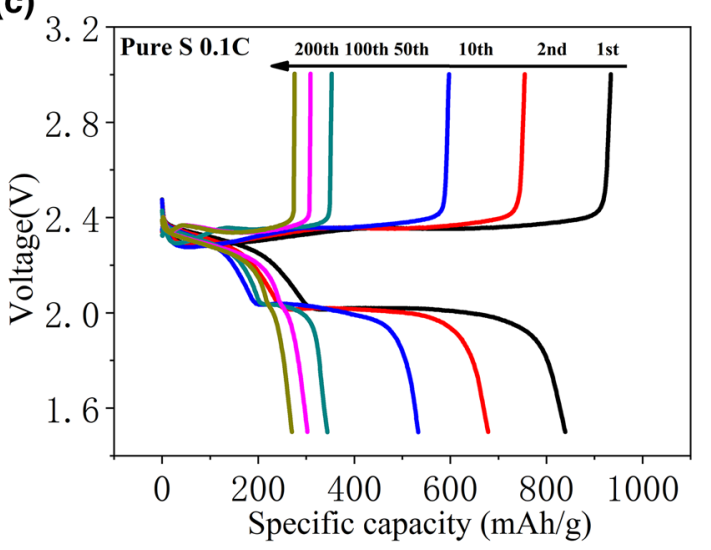

(b)

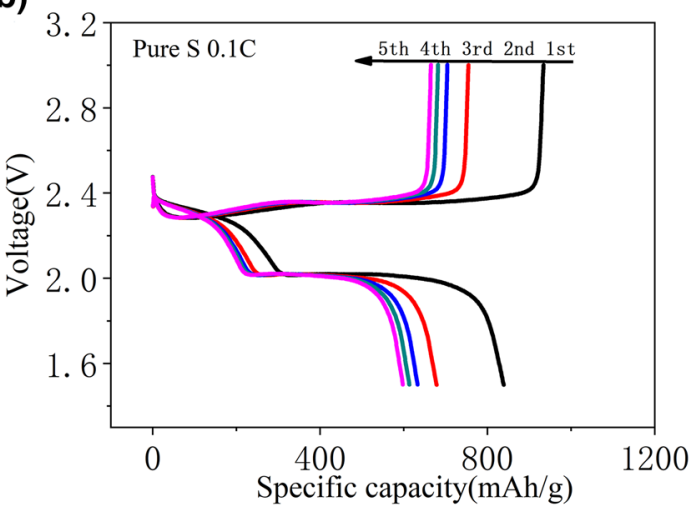

(d)

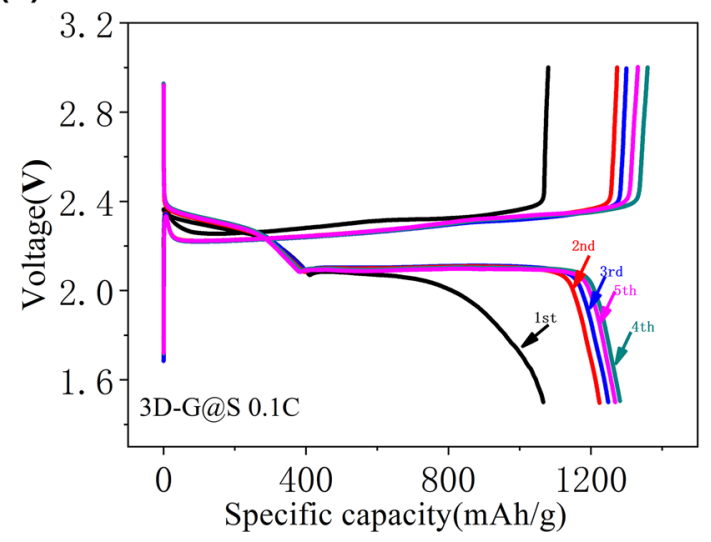

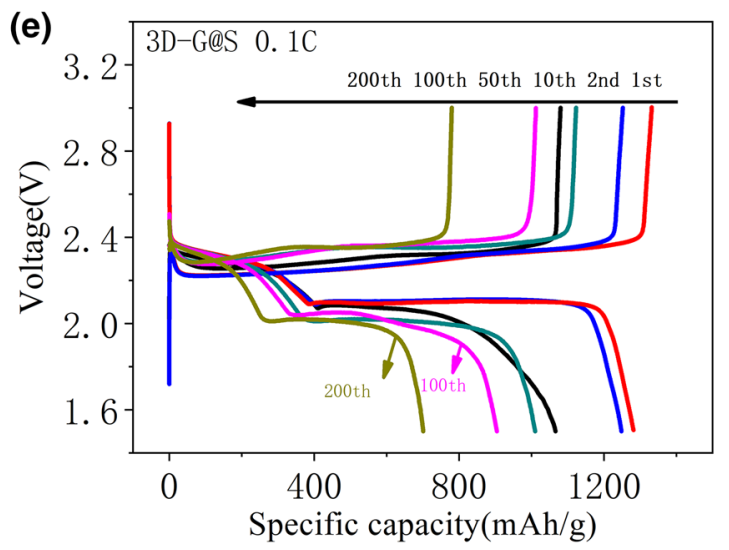

Fig. 5 a The first and second CV curves of pure S and 3D-G@S; $\mathbf{b}$ the charge/discharge curves from the first cycle to fifth cycle at $0.1 \mathrm{C}$ for pure S; c the first 200 cycles charge/discharge curves of

It is clearly shown that the cyclic performance and coulomb efficiency has been boosted after 3D-G was served as a sulfur host. Compared to pure $S$, the utilization rate of the 3D-G@S composite material was increased by $8.0 \%$ and the capacity retention ratio was increased by $13.5 \%$. Thanks to the unique 3D-G structure with high surface area as well as large pore volume, it can be served as effective pure $S$ at $0.1 \mathrm{C} ; \mathbf{d}$ the charge/discharge curves of 3D-G@S from the first cycle to fifth cycle at $0.1 \mathrm{C}$; e the first 200 cycles charge/discharge curves of 3D-G@S at $0.1 \mathrm{C}$

host for loading sulfur and enhancing their high utilization. Furthermore, graphene-like nanosheets can effectively constrain the polysulfide shuttle effect to achieve stable cyclic life. Figure $6 \mathrm{~b}$ shows the cycling stability of the 3D-G@S composite cathodes at $0.2 \mathrm{C}$ rate, the initial discharge capacity is $1182.3 \mathrm{mAh} \mathrm{g}^{-1}$ with $70.4 \%$ utilization rate of active materials. The 3D-G@S material retains 
Table 2 The redox potential of CV curves for pure S and 3D-G@S

\begin{tabular}{llll}
\hline Sample & $\begin{array}{l}\text { Oxidation } \\
\text { potential }(\mathrm{V})\end{array}$ & $\begin{array}{l}\text { Reduction } \\
\text { potential }\left(\mathrm{V}_{1}\right)\end{array}$ & $\begin{array}{l}\text { Reduction } \\
\text { potential }\left(\mathrm{V}_{2}\right)\end{array}$ \\
\hline Pure S (1st) & 2.65 & 2.00 & 2.27 \\
Pure S (2nd) & 2.50 & 2.00 & 2.28 \\
3D-G@S (1st) & 2.46 & 2.04 & 2.25 \\
3D-G@S (2nd) & 2.49 & 2.05 & 2.30 \\
\hline
\end{tabular}

the specific capacity of $539.4 \mathrm{mAh} \mathrm{g}^{-1}$ with $45.6 \%$ capacity retention ratio after 200 cycles. Figure $6 c$ demonstrates that the specific capacities at different $C$-rates of pure $S$ and 3D-G@S, respectively. The cell made from 3D-G@S shows an average discharge capacity of $1250 \mathrm{mAh} \mathrm{g}^{-1}$, $950 \mathrm{mAh} \mathrm{g}^{-1}, 720 \mathrm{mAh} \mathrm{g}^{-1}, 680 \mathrm{mAh} \mathrm{g}^{-1}$, at $0.1,0.2,0.5$, $1 \mathrm{C}$, respectively. As the current density is turned back to 0.1 from $1 \mathrm{C}$, a reversible capacity of $1000 \mathrm{mAh} \mathrm{g}^{-1}$ is

(a)

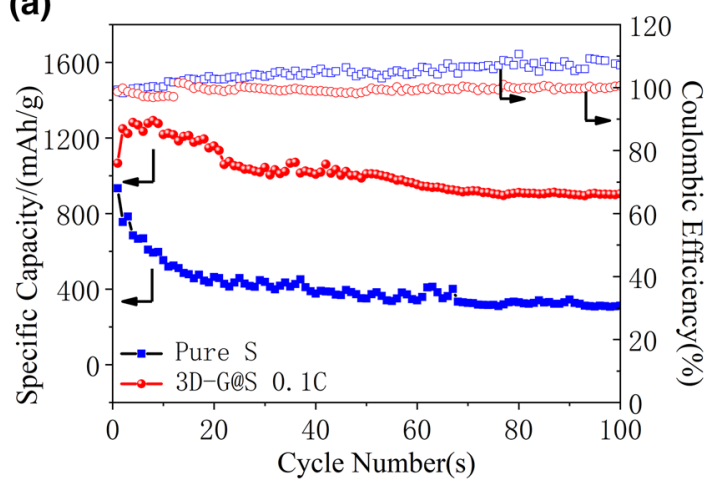

(c)

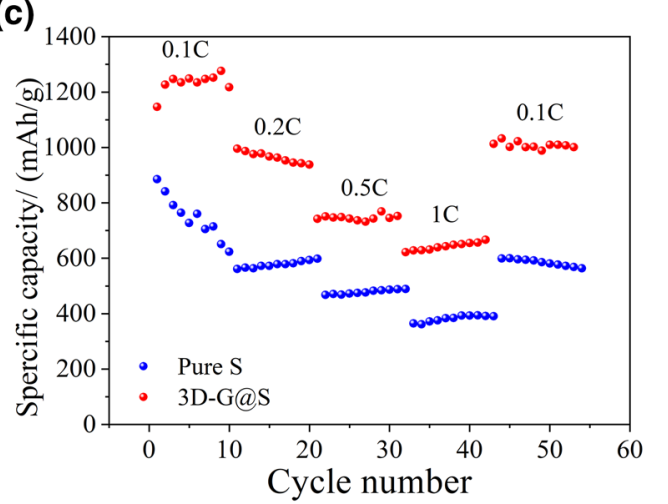

achieved, indicating good rate performance of the battery. The 3D-G@S composite electrode has higher specific capacity than pure $S$ electrode at different $C$-rates. Since the insulating pure $S$ has bad electric conductivity, the unique interconnected graphene-like structures of $3 D-G$ improve electric conductivity, and shorten the transport distance for electrons, therefore enhancing the rate capability.

Besides the cycling performance, to evaluate the effects of 3D-G structure, it is also necessary to conduct electrochemical impedance spectroscopy (EIS) to understand the reaction mechanisms during charge and discharge. Figure $6 \mathrm{~d}$ shows the Nyquist plots for pure $S$ and 3D-G@S, which consist of one depressed semicircle and one straight line in the whole frequency region. Notably, the charge transfer resistance at the interface of 3D-G@S is much smaller than pure $S$, indicating that 3D-G facilitates the charge transfer process. The equivalent circuits were (b)

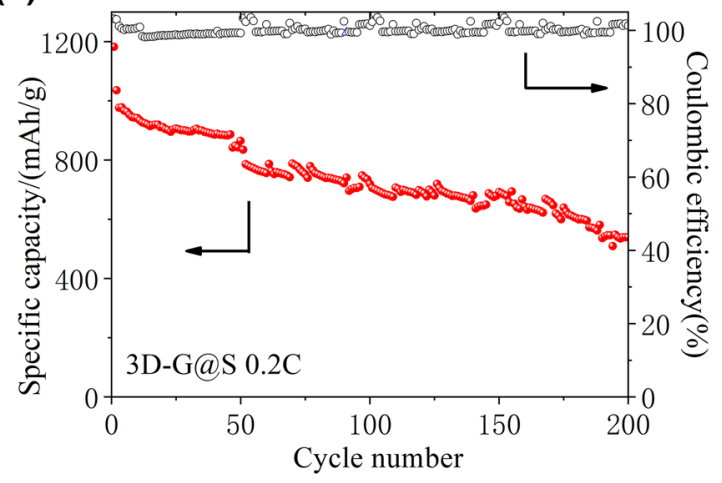

(d)

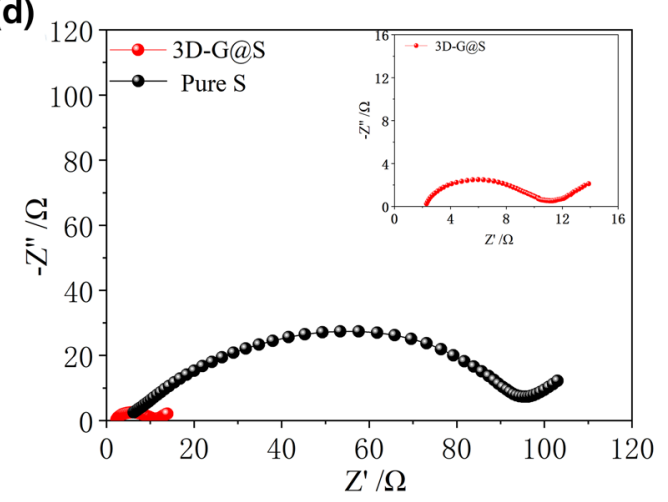

(e)

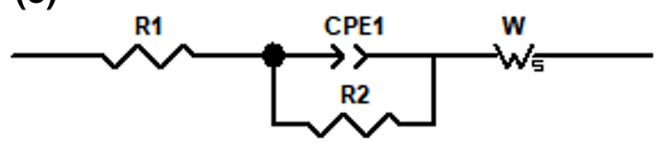

Fig. 6 a The cycle performance of 3D-G@S and Pure S at $0.1 \mathrm{C} ; \mathbf{b}$ the cycle performance of 3D-G@S at $0.2 \mathrm{C}$; $\mathbf{c}$ rate performance at various current rates $\left(1 \mathrm{C}=1675 \mathrm{~mA} \mathrm{~g}^{-1}\right)$; $\mathbf{d}$ the $A C$ impedance dia- gram of pure S and 3D-G@S before cycles. e The model of equivalent circuit before cycles; Inset in $\mathbf{d}$ shows amplified plots from the high to medium frequency range 
pictured in Fig. 6e to clarify the interface charge-transfer resistance. The $R 1$ represents a solution resistance and $R 2$ is assigned to be the charge transfer resistance. Meanwhile, the CPE1 is the impedance of double layer capacitor. Diffusion generates an impedance called Warburg impedance. Figure 6e shows that R1 and R2 of pure S electrode are 5.16 $\Omega$ and $95.56 \Omega$, respectively, compared to $1.65 \Omega$ and 11.87 $\Omega$ of 3D-G@S electrode. It is obvious that graphene-like structures on 3D-G@S composite materials have smaller charge transfer resistance to decrease the surface impedance and boost the rate capability of 3D-G@S.

\section{Conclusion}

To summarize, we report a simple, scalable fabrication process for preparing a novel graphene-wrapped porous carbon, by using low cost PAA cation-exchange resin as carbon precursor which introduces nickel ions to work as a catalyst during the carbonization process. It shows that the as prepared carbon materials possess white fungus-like structure. We consider that such a structure could endow sulfur particles with electrically conducting property, and restrict the dissolution of polysulfides. The 3D-G@S composite, as cathode materials for $\mathrm{Li}-\mathrm{S}$ batteries, showed high specific capacity and good cycling stability. Owing to their unique structure, this 3D-G can be serving as new polysulfides reservoir with improved performance of $\mathrm{Li}-\mathrm{S}$ batteries for future applications in renewable energy storage.

Acknowledgements This work is supported by the National Natural Science Foundation of China (51604086, 21673051), the Guangdong Province Science \& Technology Bureau (2016A010104015).

\section{Compliance with ethical standards}

Conflict of interest The authors declare that they have no conflict of interest.

\section{References}

1. Kong L, Jin Q, Zhang X, Li B, Chen J, Zhu W, Huang J, Zhang Q (2019) Towards full demonstration of high areal loading sulfur cathode in lithium-sulfur batteries. J Energy Chem 39:17-22

2. Li M, Lu J, Chen ZW, Amine K (2018) 30 years of lithium-ion batteries. Adv Mater 30:1800561

3. Jia XL, Nazar LF (2010) Advances in Li-S batteries. J Mater Chem 20:9821-9826

4. Kaiser MR, Han Z, Liang J, Dou SX, Wang J (2019) Energy storage materials 19:1-15. https://doi.org/10.1016/j.ensm.2019.04.001

5. Xue M, Chen C, Tan Y, Ren Z, Li B, Zhang C (2018) Mangosteen peel-derived porous carbon: synthesis and its application in the sulfur cathode for lithium sulfur battery. J Mater Sci 53:11062-11077
6. Cheng H, Cai N, Wang M (2019) Facile and scalable synthesis of micro-mesoporous carbon/magnesium oxide/sulfur composite for lithium-sulfur batteries. Solid State lonics 337:12-18

7. Kong L, Chen X, Li BQ, Peng HJ, Huang JQ, Xie J, Zhang Q (2018) A Bifunctional Perovskite Promoter for Polysulfide Regulation toward Stable lithium-sulfur Batteries. Adv Mater 30:1705219

8. Li ZQ, Yin LW (2015) Nitrogen-doped MOF-derived micropores carbon as immobilizer for small sulfur molecules as a cathode for lithium sulfur batteries with excellent electrochemical performance. ACS Appl Mater Interfaces 7:4029-4038

9. Li Z, Jiang Y, Yuan LX, Yi ZY, Wu C, Liu Y, Strasser P, Huang YH (2014) A highly ordered Meso@microporous carbon-supported sulfur@smaller sulfur core-shell structured cathode for Li-S batteries. ACS Nano 8:9295-9303

10. Schuster J, He G, Mandlmeier B, Yim T, Lee KT, Bein T, Nazar LF (2012) Spherical ordered mesoporous carbon nanoparticles with high porosity for lithium-sulfur batteries. Angew Chem Int Ed 51:3591-3595

11. Rehman S, Gu XX, Khan K, Mahmood N, Yang WL, Huang XX, Guo SJ, Hou YL (2016) 3D vertically aligned and interconnected porous carbon nanosheets as sulfur immobilizers for high performance lithium-sulfur batteries. Adv Energy Mater 6:1502518

12. Tarascon JM (2010) Key challenges in future Li-battery research. Philos Trans R Soc A Math Phys Eng Sci 368:3227-3241

13. Kaiser MR, Han Z, Wang J (2019) J Power Sources 437:226925. https://doi.org/10.1016/j.jpowsour.2019.226925

14. Seh ZW, Sun YM, Zhang QF, Cui Y (2016) Designing high-energy lithium-sulfur batteries. Chem Soc Rev 45:5605-5634

15. Wang XW, Gao T, Han FD, Ma ZH, Zhang ZA, Li J, Wang CS (2016) Stabilizing high sulfur loading $\mathrm{Li}-\mathrm{S}$ batteries by chemisorption of polysulfide on three-dimensional current collector. Nano Energy 30:700-708

16. Busche MR, Adelhelm P, Sommer H, Schneider H, Leitner $\mathrm{K}$, Janek J (2014) Systematical electrochemical study on the parasitic shuttle-effect in lithium-sulfur-cells at different temperatures and different rates. J Power Sources 259:289-299

17. Diao Y, Xie K, Xiong SZ, Hong XB (2012) Insights into Li-S battery cathode capacity fading mechanisms: irreversible oxidation of active mass during cycling. J Electrochem Soc 159:A1816-A1821

18. Diao Y, Xie K, Xiong SZ, Hong XB (2013) Shuttle phenomenonThe irreversible oxidation mechanism of sulfur active material in Li-S battery. J Power Sources 235:181-186

19. Zhang L, Guo JH (2019) Understanding the reaction mechanism of lithium-sulfur batteries by in situ/operando X-ray absorption spectroscopy. Arab J Sci Eng 44:6217-6229

20. Lu H, Chen Z, Yuan Y, Du HL, Wang JL, Liu X, Hou ZZ, Zhang K, Fang J, Qu YH (2019) A rational balance design of hybrid electrolyte based on ionic liquid and fluorinated ether in lithium sulfur batteries. J Electrochem Soc 166:A2453-A2458

21. Wang ZW, Wang SP (2019) Synergistic suppression of the shuttle effect and absorption of electrolytes using a functional rich amine porous organic polymer/acetylene black-polypropylene separator in Li-S batteries. Electrochim Acta 306:229-237

22. Xin S, Gu L, Zhao NH, Yin YX, Zhou LJ, Guo YG, Wan LJ (2012) Smaller sulfur molecules promise better lithium-sulfur batteries. J Am Chem Soc 134:18510-18513

23. Ji LW, Rao MM, Zheng HM, Zhang L, Li YC, Duan WH, Guo JH, Cairns EJ, Zhang YG (2011) Graphene oxide as a sulfur immobilizer in high performance lithium/sulfur cells. J Am Chem Soc 133:18522-18525

24. Yang X, Zhang L, Zhang F, Huang Y, Chen YS (2014) Sulfurinfiltrated graphene-based layered porous carbon cathodes for high-performance lithium-sulfur batteries. ACS Nano 8:5208-5215

25. Walle MD, Zhang Z, Zhang M, You X, Li Y, Liu Y (2018) Hierarchical 3D nitrogen and phosphorous codoped graphene/carbon 
nanotubes-sulfur composite with synergistic effect for high performance of lithium-sulfur batteries. J Mater Sci 53:2685-2696

26. Ma YF, Chen YS (2015) Three-dimensional graphene networks: synthesis, properties and applications. Nat Sci Rev 2:40-53

27. Xu H, Deng YF, Shi ZC, Qian YX, Meng YZ, Chen GH (2013) Graphene-encapsulated sulfur (GES) composites with a core-shell structure as superior cathode materials for lithium-sulfur batteries. J Mater Chem A 1:15142-15149

28. Cheng XB, Huang JQ, Zhang Q, Peng HJ, Zhao MQ, Wei F (2014) Aligned carbon nanotube/sulfur composite cathodes with high sulfur content for lithium-sulfur batteries. Nano Energy 4:65-72

29. Liu J, Liu B, Wang CW, Huang ZX, Hu LC, Ke X, Liu LY, Shi ZC, Guo ZP (2017) Walnut shell-derived activated carbon: synthesis and its application in the sulfur cathode for lithium-sulfur batteries. J. Alloys Compd. 718:373-378

30. Han SC, Song MS, Lee H, Kim HS, Ahn HJ, Lee JY (2013) Effect of multiwalled carbon nanotubes on electrochemical properties of lithium/sulfur rechargeable batteries. J Electrochem Soc 150:A889-A893

31. Xiao LF, Cao YL, Xiao J, Schwenzer B, Engelhard MH, Saraf LV, Nie ZM, Exarhos GJ, Liu J (2012) A soft approach to encapsulate sulfur: polyaniline nanotubes for lithium-sulfur batteries with long cycle life. Adv Mater 24:1176-1181

32. Zhang YG, Zhao Y, Konarov A, Li Z, Chen P (2015) Effect of mesoporous carbon microtube prepared by carbonizing the poplar catkin on sulfur cathode performance in $\mathrm{Li} / \mathrm{S}$ batteries. J. Alloys Compd. 619:298-302

33. Zheng GY, Yang Y, Cha JJ, Hong SS, Cui Y (2011) Hollow carbon nanofiber-encapsulated sulfur cathodes for high specific capacity rechargeable lithium batteries. Nano Lett 11:4462-4467

34. PółrolniczakP Nowicki $P$, Wasiński K, Pietrzak R, Walkowiak M (2016) Biomass-derived hierarchical carbon as sulfur cathode stabilizing agent for lithium-sulfur batteries. Solid State lonics 297:59-63

35. Kaiser MR, Liang X, Konstantinov K, Liu H-K, Dou S-X, Wang J-Z (2015) Chem Eur J 21:10061-10069. https://doi.org/10.1002/ chem.201500429

36. Kaiser MR, Ma Z, Wang X, Han F, Gao T, Fan X, Wang J, Liu HK, Dou SX, Wang CS (2017) ACS Nano 11(9):9048-9056. https:// doi.org/10.1021/acsnano.7b03591

37. Zhang CF, Wu HB, Yuan CZ, Guo ZP, Lou XW (2012) Confining sulfur in double-shelled hollow carbon spheres for lithium-sulfur batteries. Angew Chem Int Ed 51:9592-9595

38. Zhou GM, Zhao YB, Manthiram A (2015) Dual-confined flexible sulfur cathodes encapsulated in nitrogen-doped double-shelled hollow carbon spheres and wrapped with graphene for $\mathrm{Li}-\mathrm{S}$ batteries. Adv. Energy Mater. 5:1402263

39. Li XS, Cai WW, Colombo L, Ruoff RS (2019) Evolution of graphene growth on $\mathrm{Ni}$ and $\mathrm{Cu}$ by carbon isotope labeling. Nano Lett 9:4268-4272

40. Eizenberg M, Blakely JM (1979) Carbon interaction with nickel surfaces: monolayer formation and structural stability. J Chem Phys 71:3467-3477

41. Zhu Y, Xu GY, Zhang XL, Wang SJ, Li C, Wang GX (2017) Hierarchical porous carbon derived from soybean hulls as a cathode matrix for lithium-sulfur batteries. J Alloys Compd 695:2246-2252

Publisher's Note Springer Nature remains neutral with regard to jurisdictional claims in published maps and institutional affiliations. 